\title{
Time-dependent correlations for spin Van der Waals systems
}

\author{
Raf Dekeyser \\ Instituut voor Theoretische Fysika, Katholieke Universiteit Leuven, B-3030 Heverlee, Belgium \\ M. Howard Lee \\ Department of Physics, University of Georgia, Athens, Georgia 30602 \\ (Received 3 May 1978)
}

\begin{abstract}
The time-dependent autocorrelation function has been derived for the $x$ component of the total spin for the $S=1 / 2$ constant-coupling anisotropic Heisenberg model, i.e., the Van der Waals system. For $T>T_{c}$, the time correlation is shown to be Gaussian for both $X Y$-like and Ising-like regimes of the model. For $T<T_{c}$, the correlation function is still Gaussian if the model is $X Y$-like; but it is oscillatory if the model is Isinglike. Critical slow down appears only with the Ising-like system for this time-correlation function.
\end{abstract}

\section{INTRODUCTION}

By a spin Van der Waals system, we mean a system of lattice spins each of which is coupled to all other spins with equal strength. Such a system may be thought of as the high-density limit of an anisotropic Heisenberg model. Van der Waals systems belong to the class of mean-field models and are analytically soluble. Although limited in scope, the exact solutions for this class of systems can be useful in providing an insight into more complex systems. Recently, these mean-field models have taken on an added significance through the discovery that certain metal hydrides exhibit mean-field critical behavior. ${ }^{1}$ The static properties of spin Van der Waals systems have been extensively studied by Kittel and Shore,$^{2}$ Niemeijer, ${ }^{3}$ and Vertogen and DeVries. ${ }^{4}$ They proved that equilibrium thermodynamic properties of spin Van der Waals systems are independent of spin dimensionality. That is, the isotropic Heisenberg and Ising systems in the high-density limit have identical equilibrium thermodynamic behavior. ${ }^{5}$

To our knowledge the dynamic properties of spin Van der Waals systems are not yet well known. It would be interesting to learn exactly how the Van der Waals systems respond to a time-dependent perturbation and dissipate it in time. It would also be. of interest to learn whether spin Van der Waals systems all have dynamically equivalent behavior, or whether they all belong to different dynamic universality classes. ${ }^{6}$ For these pur poses, we shall consider the $S=\frac{1}{2}$ constant-coupling anisotropic Heisenberg model and study the timecorrelation function in appropriate physical regimes. ${ }^{7}$ To obtain the time-correlation function, it is necessary to resolve the time-dependent part (by solving the Heisenberg equation of motion) and then to evaluate various quantities which do not depend on time. We shall study the transverse component of the time-correlation function $S^{x x}(t)$ $=\left\langle S_{x}(t) S_{x}(0)\right\rangle$, where $S_{x}$ is the $x$ component of the total spin. Since $S_{x}$ (or $S_{y}$ ) is not a constant of motion for the anisotropic Heisenberg model [see Eq. (2)], $S_{x}(t)$ has a nontrivial time evolution. On the other hand $S_{z}$ is a constant of motion. Hence, the longitudinal component of the time-correlation function $\mathrm{S}^{z z}$ is trivial. It should be remembered that in this paper we discuss the transversecorrelation function only (whether the anisotropy is $X Y$-like or Ising-like).$^{8}$

For the one-dimensional nearest-neighbor $X Y$ model, the time-correlation function $S^{x x}(t)$ at the high-temperature limit is now known. Sur et al., ${ }^{9}$ Brandt and Jacoby, ${ }^{10}$ and Capel and Perk ${ }^{11}$ have shown that it is Gaussian. Since there is no phase transition in the one-dimensional nearest-neighbor $X Y$ model, the high-temperature form for the time-correlation function may be expected to persist, if in some modified way, into lower temperatures. We find that there seems to exist an interesting but evidently coincidental connection between this result for the one-dimensional nearestneighbor $X Y$ model and our result for the constantcoupling $X Y$ model.

In Sec. II, our spin Van der Waals model is briefly described. In Sec. III, we review the technique of calculating the static quantities in the thermodynamic limit. In Sec. IV, the time-correlation function is derived for different physical regimes and also for different anisotropic limits. In Sec. V, we make a few concluding remarks.

\section{DESCRIPTION OF THE MODEL}

Consider a system of $N \frac{1}{2}$ spins, where $N$ is considered to be a large number (the thermodynamic limit is always implied in our analysis). The components of the individual spin operator at site $i$ are denoted by $S_{i}^{x} ; S_{i}^{y}$, and $S_{i}^{z}, 1 \leqslant i \leqslant N$, and those of 
the total spin by $S_{x}, S_{y}$, and $S_{z}$, e.g.,

$$
S_{x}=\sum_{i \neq 1}^{N} S_{i}^{x}
$$

In this system all spins are assumed to interact pairwise and with equal strength, independent of their relative distances. The interaction, however, is assumed to be anisotropic with respect to the spin components. With a conveniently chosen additive constant, the Hamiltonian of this system is given as follows:

$$
\begin{aligned}
\mathcal{H} & =-\frac{1}{N} \sum_{i \neq j}\left[J\left(S_{i}^{x} S_{j}^{x}+S_{i}^{y} S_{j}^{y}\right)+J_{z} S_{i}^{z} S_{i}^{z}\right]-\frac{1}{4}\left(2 J+J_{z}\right) \\
& =-\frac{1}{N}\left(J \vec{S} \cdot \overrightarrow{\mathrm{S}}-\lambda S_{z}^{2}\right)
\end{aligned}
$$

where

$$
\overrightarrow{\mathrm{S}}=\left(S_{x}, S_{y}, S_{z}\right)
$$

and

$$
\lambda=J-J_{z} ; \quad J>0, \quad J_{z}>0 .
$$

We observe that the Hamiltonian is diagonal, i.e., with $\vec{S}^{2}=S(S+1), \mathcal{H}=f\left(S, S_{z}\right)$ only. Now $\lambda$ can be either positive or negative. If $\lambda>0$, the model is $X Y$-like; and if $\lambda<0$, it is Ising-like. We shall not consider the isotropic Heisenberg case $(\lambda=0)$ since all the components of the total spin, being conserved quantities, have trivial dynamic properties. The static properties of this system are not our main interest. But we shall begin by describing them first, since dynamic quantities must ultimately be expressed in terms of time-independent quantities.

\section{STATIC PROPERTIES}

The eigenstates of Hamiltonian (2) may be characterized by a set of quantum numbers $\left\{S\right.$ and $\left.S_{z}\right\}$. Evidently these states are multiply degenerate. The degeneracy must be accounted for in summing over states in evaluating, e.g., the partition function. For convenience we assume $N$ to be an even number. The degeneracy $g$ can be expressed as the difference between two binomial coefficients

$$
g\left(S, S_{z}\right)=g(S)=\left(\begin{array}{c}
N \\
\frac{1}{2} N-S
\end{array}\right)-\left(\begin{array}{c}
N \\
\frac{1}{2} N-S-1
\end{array}\right),
$$

where $\frac{1}{2} N \geqslant S \geqslant 0 .{ }^{12}$ The above expression can be approximated for large $N$ as

$$
g(S)=2^{N+1}[s /(1+s)] e^{-N W(s)},
$$

where

$$
s=2 S / N
$$

$$
\begin{aligned}
W(s) & =\frac{1}{2}[(1-s) \ln (1-s)+(1+s) \ln (1+s)] \\
& =2(S / N)^{2}+\frac{4}{3}(S / N)^{4}+\cdots .
\end{aligned}
$$

It may be recognized that the degeneracy factor $g(S)$ represents the number of possible equivalent spin configurations (i.e., configurations having the same energy) for a given value of $S .{ }^{13}$ Thus, $\ln g(S)$ is related to the entropy of the system, ${ }^{14}$ and the free energy $F=\mathfrak{H}-T S_{e}$ has a minimum at a certain value of the total spin $S$. The partition function may be evaluated in the following way:

$$
\begin{aligned}
Z & =\operatorname{Tr} e^{-\beta \Re} \\
& =\sum_{S=0}^{N / 2} \sum_{S_{z}}^{S} g(S) e^{\beta J S(S+1) / N-\beta \lambda s_{z}^{2} / N} .
\end{aligned}
$$

Substituting for $g(S)$, we obtain

$$
=2^{N+1} \sum_{S}\left(\frac{2 S / N}{1+2 S / N}\right) e^{N G(s)},
$$

where

$$
\begin{aligned}
G(S) & =-W(S)+\frac{\beta J S(S+1)}{N^{2}}+\frac{1}{N} \ln \sum_{S_{z}} e^{-\beta \lambda s_{z}^{2} / N} \\
& \approx-(2-\beta J)\left(\frac{S}{N}\right)^{2}+\cdots+\frac{1}{N} \ln \sum_{S_{z}} e^{-\beta \lambda s_{z}^{2} / N} .
\end{aligned}
$$

We observe that $-\beta^{-1} G(S)$ is the free energy per spin for a fixed value of the total spin $S$. Evidently the phase factor $G(S)$ is the most important factor in determining the sum in Eq. (10). The dominant contributions come from the maximum of $G(S)$ given at some value of $S=S_{0}$. Hence, it will be found sufficient to evaluate the sum about $S_{0}$, where $G\left(S_{0}\right)=G_{\max }{ }^{15}$ Now $S_{0}$ must be a macroscopic number, i.e., $S_{0}=f(N)$ in order that the prefactor of $e^{N G(s)}$ in the partition sum [the term inside the parentheses in Eq. (10)] does not vanish when evaluated about $S_{0}$. We shall find $S_{0}$ to be related to the long-range order of the system.

For both the $X Y$-like and Ising-like systems there are two general classes which need be distinquished: high- and low-temperature regimes. For high temperatures, however, the $X Y$ and Ising systems are physically very similar so that they may be treated together. Our mathematical solutions for the partition function (given later in Sec. III) have a simple physical basis. This basis is first briefly discussed below and, we believe, will make the ensuing mathematical analysis rather transparent.

At high temperatures (i.e., $\beta J \ll 2$ ), the interaction energy $J S(S+1) / N^{2}$ is small compared with $k T$. Hence, the free energy or $-G$ is determined largely by the entropy term $-W$ [see Eq. (11a)]. At a fixed high temperature, the maximum entropy

and 
(i.e., minimum $F$ or maximum $G$ ) results from the state of $N \frac{1}{2}$ spins which are maximally disordered, i.e., $S_{0} \neq O(N)$, so that $S_{0} / N \rightarrow 0$ as $N \rightarrow \infty .{ }^{16}$ For both the $X Y$ and Ising systems we shall see that $S_{0}=O\left(N^{1 / 2}\right)$ confirming what is already known about the absence of long-range order at high temperatures.

At low temperatures (i.e., $\beta J \gg 2$ ), the interaction energy $J S(S+1) / N^{2}$ is now large compared with $k T$ and it competes with the entropy in determining $G$. An ordered state will decrease the contribution of the entropy to $G$; but it can still increase $G$ through the interaction energy, which of course increases with the ordering of spins. A maximum $G$ can result from a such macroscopically ordered state of $N \frac{1}{2}$ spins, i.e., $S_{0}=O(N)$ so that $S_{0} / N=O(1)$ as $N \rightarrow \infty$. This implies, of course, that there is a condensed phase for $\beta J$ $\geqslant \beta_{c} J$ for some $\beta_{c}$, to be later determined.

\section{A. High-temperature $X Y$ and Ising solutions}

When $\beta=1 / k T$ is small, the phase factor of Eq. (10) is dominated by the entropy term $-W$. Since large values of $S$ give little contribution to the partition function, we may approximate $W(S)$ by its first term in the series expansion, giving the following Gaussian form:

$$
\begin{aligned}
Z=2^{N+1} \sum_{S=0}^{N / 2} & \left(\frac{2 S}{N}\right) e^{-(2-\beta J) s^{2} / N} \\
& \times \sum_{s_{z}=-S}^{S} e^{-\beta \lambda s_{z}^{2} / N} .
\end{aligned}
$$

The maximum of the $S$ summand of Eq. (12) is attained when $S=S_{0}=O\left(N^{1 / 2}\right)$. Thus, replacing the sums in Eq. (12) by integrals over $S=N^{1 / 2} x$ and $S_{z}=N^{1 / 2} z$, we have

$$
\begin{aligned}
Z=2^{N+1} N^{1 / 2} \int_{0}^{\infty} & d x e^{-(2-\beta J) x^{2}} \\
& \times 2 x \int_{-x}^{x} d z e^{-\beta \lambda z^{2}},
\end{aligned}
$$

where the upper limit on $x$ has been extended to $\infty$ introducing little error. The above integral can be directly integrated by parts giving

$$
Z=2^{N+1}(\pi N)^{1 / 2}(2-\beta J)^{-1}\left(2-\beta J_{z}\right)^{-1 / 2} .
$$

The leading correction to this expression comes from the neglected $(1+s)^{-1}$ [see Eq. (10)] and the correction will be a factor $N^{1 / 2}$ smaller than the above term in Eq. (14). The specific heat vanishes for $T$ above $T_{c}$ as it must for a mean-field model. ${ }^{17}$

Observe that our result [Eq. (14)] is independent of $\lambda$ positive or negative. It must be remembered, however, that for the $X Y$-like system the above result applied with $J>J_{z}$, whereas for the Isinglike system $J_{z}>J$. To show how the two different conditions affect the result, we derive the fluctuations. For Van der Waals systems, the high-temperature spin fluctuations are given by ${ }^{18}$

$$
\begin{aligned}
& Y^{x x}=\sum_{r=1}^{N}\left\langle S_{0}^{x} S_{r}^{x}\right\rangle=\frac{1}{2} \frac{\partial}{\beta \partial J} \ln Z, \\
& Y^{z z}=\sum_{r=1}^{N}\left\langle S_{0}^{z} S_{r}^{z}\right\rangle=\frac{\partial}{\beta \partial J_{z}} \ln Z .
\end{aligned}
$$

Thus, it follows directly from Eqs. (14) and (15) that, for the $X Y$-like system, $Y^{x x}=\frac{1}{2}\left(\beta_{c} J-\beta_{c}\right)^{-1}$ and $Y^{z z}=\frac{1}{2}\left(\beta_{c} J-\beta J_{z}\right)^{-1}$, and that for the Ising-like system, $Y^{x x}=\frac{1}{2}\left(\beta_{c} J_{z}-\beta J\right)^{-1}$ and $Y^{z z}=\frac{1}{2}\left(\beta_{c} J_{z}-\beta J_{z}\right)^{-1}$. That is, for the $X Y$-like system, $Y^{x x}$ is divergent but $Y^{z z}$ is nondivergent at $T_{c}$; whereas, for the Ising-like system, the reverse holds. We have used here the results shown later in Sec. III that the $X Y$-like system has $\beta_{c} J=2$ and the Ising-like system has $\beta_{c} J_{z}=2$.

\section{B. Low-temperature $X Y$ solutions}

For $\lambda$ positive (i.e., $X Y$ regime), the $S_{z}$ dependence in the partition function [see Eq. (12)] remains Gaussian for all $\beta$ and the main contributions still come from $S_{z}=O\left(N^{1 / 2}\right)$ or less. But for $\beta J<\beta_{c} J$ $=2$, the $S$ dependence is not Gaussian, i.e., the previous high-temperature approximation for $W(S)$ is no longer valid. An examination shows that the phase factor $G(S)$ [see Eq. (10)] has a sharp maximum at $S=S_{0}=O(N)$, so that $\exp N G(S)$ is sharply peaked at $S=S_{0}$. The condition $G^{\prime}\left(S_{0}\right)=0$ gives the familiar mean-field equation for the long-range order of the system, ${ }^{13}$

$$
-W^{\prime}+\beta J S_{0} / N=0
$$

or

$$
S_{0} / N=\frac{1}{2} \tanh \beta J S_{0} / N \text {. }
$$

In the usual way, nontrivial solutions exist for $T$ below $T_{c}=J / 2 K$ if $S_{0}=O(N)$. Hence, defining $\sigma$ $=2 S_{0} / N$, we may rewrite Eq. $(16 \mathrm{~b})$ as

$$
\sigma=\tanh \left(\beta / \beta_{c}\right) \sigma
$$

with $\beta_{c} J=2 .{ }^{19}$ Observe that Eq. (16c) is independent of $J_{z}$ as long as $\lambda$ is positive (i.e., $J>J_{z}$ ). That is, the critical temperature $\beta_{c} J=2$ is universal for the Van der Waals $X Y$-like system.

One can now expand $G(S)$ in Eq. (10) about $S_{0}$ and carry out the $S$ integration by substituting $S=S_{0}$ $+N x$. The $S_{z}$ integration may be kept as before. Also since the two exponential functions are peaked at different regions with little overlap, the limits of integration in both cases may be extended. Thus we have 


$$
\begin{aligned}
Z & =g\left(S_{0}\right) e^{\beta J S_{0}^{2} / N} \int_{-\infty}^{\infty} d x e^{-\beta N B x^{2}} \int_{-\infty}^{\infty} d z e^{-\beta \lambda z^{2}} \\
& =g\left(\frac{1}{2} N \sigma\right) e^{\beta J N \sigma^{2} / 4} N \pi(\beta \lambda B)^{-1 / 2}\left[1+O\left(N^{-1}\right)\right],
\end{aligned}
$$

where

$$
B=\left[2 /\left(1-\sigma^{2}\right)\right]-\beta J \approx\left(\beta-\beta_{c}\right) J \text { as } \beta-\beta_{c}+.
$$

The specific heat follows directly from Eq. (17a) giving the usual mean-field result, in particular, $C / N=3 k$ at $T=T_{c}$

To calculate the fluctuations in the long-range order, it is necessary to include the corrections of order $N^{-1}$ in the partition function. The corrections may be obtained by retaining second-order terms in the expansion of the $S$ integrand about $S_{0} \cdot{ }^{15}$ The partition function valid to the order $N^{-1}$ is as follows:

$$
Z=Z_{0}\left[1+Z_{1} N^{-1}+O\left(N^{-2}\right)\right],
$$

where $Z_{0}$ denotes the partition function given by Eq. (17a) and

$$
\begin{aligned}
Z_{1}= & \frac{1}{2 B}\left(\frac{(\beta J)^{2}}{2}+\frac{2 \beta J}{\sigma(1+\sigma)}-\frac{4}{\sigma(1+\sigma)^{2}}\right) \\
& -1 / B^{2}\left(\frac{5-4 \sigma+3 \sigma^{2}+2 \beta J \sigma\left(1-\sigma^{2}\right)}{\left(1-\sigma^{2}\right)^{3}}\right) \\
& +\frac{20}{3 B^{3}} \frac{\sigma}{\left(1-\sigma^{2}\right)^{4}} .
\end{aligned}
$$

\section{Low-temperature Ising solutions}

For negative $\lambda$ (i.e., $J_{z}>J$ ), the sign of the phase factor $-\lambda \beta S_{z}^{2}$ changes. Thus, the $S_{z}$ summation [see Eq. (10)] becomes very strongly dependent on its limits $(-S, S)$ so that, unlike in the $X Y$ system, it can influence the value of $S_{0}$ (the maximum of the phase factor $G(S)$ ). Introducing $\lambda=-\lambda^{\prime}$, where $\lambda^{\prime}$ is a positive quantity, we express the $S_{z}$ sum or integral in terms of Dawson's integral

$$
\begin{aligned}
\int_{-s}^{s} d S_{z} e^{\beta \lambda^{\prime} s_{z^{\prime N}}^{2}} & =2 e^{\beta \lambda^{\prime} s^{2} / N} \\
& \times\left(\frac{N}{\beta \lambda^{\prime}}\right)^{1 / 2} D\left[S\left(\frac{\beta \lambda^{\prime}}{N}\right)^{1 / 2}\right],
\end{aligned}
$$

where Dawson's integral is defined as $^{20}$

$$
D(y)=e^{-y^{2}} \int_{0}^{y} d u e^{u^{2}},
$$

which is related to erfi $y$. We note that the argument of Dawson's integral $y=\left(\beta \lambda^{\prime} / N\right)^{1 / 2} S$ can be large if $S=O(N)$. Since it is sufficient to expand $G(S)$ about $S=S_{0}$, one may thus replace $D(y)$ by its asymptotic form [assuming of course that $S_{0}=O(N)$ ]. The asymptotic expansion of $D(Y)$ is as follows:

$$
D(y)=\sum_{n=0}^{\infty} \frac{2 n !}{n !}(2 y)^{-2 n-1}=\frac{1}{2 y}+\frac{1}{4 y 3}+\cdots
$$

for $y$ large. ${ }^{20}$ Hence, we obtain for the phase factor $G(S)$,

$$
\begin{aligned}
G(S) & =-W+\beta J(S / N)^{2}+\beta \lambda^{\prime}(S / N)^{2} \\
& =-W+\beta J_{z}(S / N)^{2},
\end{aligned}
$$

which is the same expression as that for the $X Y$ system with $J_{z}$ replacing $J$. The condition that $G^{\prime}\left(S_{0}\right)=0$ gives the same mean-field equation as Eq. (16c), with $J_{z}$ replacing $J$,

$$
\sigma=\tanh \left(\beta / \beta_{c}\right) \sigma
$$

but now with $\beta_{c} J_{z}=2$. Thus, if $J_{z}>J$, the meanfield Eq. (23) is independent of $J$. That is, all Van der Waals Ising-like systems have the same value for $T_{c}{ }^{21}$

The evaluation of the partition function follows the same procedure as that for the low-temperature $X Y$ system and we obtain,

$$
\begin{aligned}
Z= & g\left(\frac{1}{2} N \sigma\right) e^{\beta J_{z} N \sigma^{2} / 4} \frac{2 N}{\beta \lambda^{\prime} \sigma} \int_{-\infty}^{\infty} d x e^{-N B z_{z} x^{2}} \\
= & g\left(\frac{1}{2} N \sigma\right) e^{\beta J_{Z} N \sigma^{2} / 4} \frac{2}{\beta \lambda^{\prime} \sigma} \\
& \times\left(\frac{N \pi}{B_{z}}\right)^{1 / 2}\left[1+O\left(N^{-1}\right)\right]
\end{aligned}
$$

where

$$
B_{z}=2 /(1-\sigma)-\beta J_{z} .
$$

If the correction terms are included, the partition function is

$$
Z=Z_{0}\left[1+Z_{1} N^{-1}+O\left(N^{-2}\right)\right],
$$

where $Z_{0}$ is given by Eq. (24a) and we have

$$
\begin{aligned}
Z_{1}= & \frac{1}{2 B_{z}}\left(\frac{(\beta J)^{2}}{2}-\frac{2 \beta J}{1+\sigma}+\frac{4}{(1+\sigma)^{2}}\right)-\frac{1}{B_{z}^{2}} \\
& \times\left(\frac{1-4 \sigma+7 \sigma^{2}}{\left(1-\sigma^{2}\right)^{3}}+\frac{2 \beta J \sigma}{\left(1-\sigma^{2}\right)^{2}}\right) \\
& +\frac{2}{\beta \lambda^{\prime} \sigma^{2}}+\frac{20}{3 B_{z}^{3}} \frac{\sigma}{\left(1-\sigma^{2}\right)^{4}} .
\end{aligned}
$$

All standard static quantities of interest can be readily obtained from our results for the partition function. In deriving the time-correlation function, it will be found necessary to evaluate still other static correlation functions such as $\left\langle S_{z}^{2 n}\right\rangle$ and $\left\langle S_{z}^{2 n} S_{x}^{2}\right\rangle$ where $n$ is any integer. These shall be treated at appropriate places in Sec. IV. 


\section{TIME-CORRELATION FUNCTIONS}

For the Van der Waals Hamiltonian Eq. (2), the time evolution of the total spin $\vec{S}$ may be obtained from the following set of Heisenberg equations of motion:

$$
\begin{aligned}
& \dot{S}_{x}=-\omega_{N}\left(2 S_{z} S_{y}+i S_{x}\right), \\
& \dot{S}_{y}=\omega_{N}\left(2 S_{z} S_{x}-i S_{y}\right), \\
& \dot{S}_{z}=0,
\end{aligned}
$$

where $\omega_{N}=\lambda / \hbar N$. These equations may be combined to give second-order differential equations separately for $S_{x}$ and $S_{y}$. They are then readily solved to give 22

$$
\begin{aligned}
& S_{x}(t)=\left[\cos \left(2 \omega_{N} t S_{z}\right) S_{x}-\sin \left(2 \omega_{N} t S_{z}\right) S_{y}\right] e^{-i \omega_{N} t}, \\
& S_{y}(t)=\left[\cos \left(2 \omega_{N} t S_{z}\right) S_{y}+\sin \left(2 \omega_{N} t S_{z}\right) S_{x}\right] e^{-i \omega_{N} t}, \\
& S_{z}(t)=S_{z},
\end{aligned}
$$

where $S_{x}=S_{x}(t=0)$, etc.

The time evolutions of $S_{x}$ and $S_{y}$ appear to be oscillatory. In fact, however, they are rather complex owing to the presence of $S_{z}$, which is a constant in time but still an operator. Thus one must maintain the order of these noncommuting operators. Using the above results, we shall deduce the time-correlation function $\left\langle S_{x}(t) S_{x}(0)\right\rangle$ for the $X Y$-like and Isinglike systems and study the time-dependent behavior for $T$ above and below $T_{c}$ for each of these systems. This time-correlation function is particularly useful since one can obtain from it the dynamic form factor or spectral density function, hence all other important dynamic quantities such as the relaxation function and dynamic susceptibility. ${ }^{23}$

For convenience we shall absorb the trivial exponential factor which appears in Eq. (27) in the definition of our time-correlation function by defining it as

$$
S^{x x}(t)=e^{i \omega_{N} t}\left\langle S_{x}(t) S_{x}(0)\right\rangle,
$$

which satisfies time-reversal symmetry. Using our results given above we obtain

$$
S^{x x}(t)=\left\langle\cos \left(2 \omega_{N} t S_{z}\right) S_{x}^{2}-\sin \left(2 \omega_{N} t S_{z}\right) S_{y} S_{x}\right\rangle .
$$

Now since $S_{z}$ is diagonal with respect to the eigenvectors of the Hamiltonian, we observe that, in the second term in Eq. (29), only the diagonal part of $S_{y} S_{x}$ can contribute in the ensemble average. Hence, we can reduce Eq. (29) to the following:

$$
S^{x x}(t)=\left\langle\cos \left(2 \omega_{N} t S_{z}\right) S_{x}^{2}\right\rangle+\frac{1}{2} i\left\langle\sin \left(2 \omega_{N} t S_{z}\right) S_{z}\right\rangle,
$$

which by expanding the trigonometric functions leads to

$$
\begin{aligned}
= & \sum_{n=0}^{\infty}(-)^{n} \frac{\left(2 \omega_{N} t\right)^{2 n}}{2 n !}\left\langle S_{z}^{2 n} S_{x}^{2}\right\rangle \\
& +\frac{1}{2} i \sum_{n=0}^{\infty}(-)^{n} \frac{\left(2 \omega_{N} t\right)^{2 n+1}}{(2 n+1) !}\left\langle S_{z}^{2 n+2}\right\rangle .
\end{aligned}
$$

Taking advantage of the $X Y$ symmetry, i.e.,

$$
\left\langle S_{z}^{2 n} S_{x}^{2}\right\rangle=\frac{1}{2}\left(\left\langle S_{z}^{2 n} S^{2}\right\rangle-\left\langle S_{z}^{2 n+2}\right\rangle\right),
$$

we finally obtain

$$
\begin{aligned}
2 S^{x x}(t)= & \sum_{n=0}(-)^{n} \frac{\left(2 \omega_{N} t\right)^{2 n}}{2 n !}\left\langle S_{z}^{2 n} S^{2}-S_{z}^{2 n+2}\right\rangle \\
& +i \sum_{n=0}(-)^{n} \frac{\left(2 \omega_{N} t\right)^{2 n+1}}{(2 n+1) !}\left\langle S_{z}^{2 n+2}\right\rangle .
\end{aligned}
$$

Observe that the time part has now been completely resolved from time-independent-correlation functions. To obtain the time-correlation function $S^{x x}(t)$, one needs to know the two equilibrium correlation functions $\left\langle S_{z}^{2 n} S^{2}\right\rangle$ and $\left\langle S_{z}^{2 n}\right\rangle$ only. These equilibrium quantities depend on $\lambda$ and also on the temperature. They may be evaluated in the manner of the partition function shown in Sec. III. We shall consider them in three different regimes: high-temperature $X Y$-like and Ising-like, lowtemperature $X Y$-like, and low-temperature Isinglike.

\section{A. High-temperature $X Y$ and Ising models}

We shall evaluate $\left\langle S_{z}^{2 n}\right\rangle$ and $\left\langle S_{z}^{2 n} S^{2}\right\rangle$ and also a few derivative terms of interest closely following the static analysis previously given (see Sec. III A). For any integer $n$ (including $n=0$ ),

$$
\begin{aligned}
\left\langle S_{z}^{2 n}\right\rangle= & Z^{-1} \sum_{S} \sum_{S_{z}} g(S) S_{z}^{2 n} e^{-\beta H\left(S, s_{z}\right)} \\
= & Z^{-1} 2^{N+1} N^{n+1 / 2} \int_{0}^{\infty} d x e^{-(2-\beta J) x^{2}} \\
& \times 2 x \int_{-x}^{x} d z z^{2 n} e^{-\beta \lambda z^{2}} \\
= & \frac{2 n !}{n !}\left(\frac{N}{4\left(2-\beta J_{z}\right)}\right)^{n}
\end{aligned}
$$

Observe that

$$
\left\langle S_{z}^{2}\right\rangle=\frac{1}{2} N /\left(2-\beta J_{z}\right),
$$

which for the pure $X Y$ model becomes independent of the temperature, $\left\langle S_{z}^{2}\right\rangle=\frac{1}{4}$ for $T>T_{c}$. Similarly we have 


$$
\begin{aligned}
\left\langle S_{z}^{2 n} S^{2}\right\rangle= & Z^{-1} 2^{N+1} N^{n+3 / 2} \int_{0}^{\infty} d x e^{-(2-\beta J) x^{2}} \\
& \times 2 x^{3} \int_{-x}^{x} d z e^{-\beta \lambda z^{2} z^{2 n}} \\
= & N\left(\frac{1}{2-\beta J}+\frac{1}{2} \frac{2 n+1}{2-\beta J_{z}}\right) \frac{2 n !}{n !}\left(\frac{N}{4\left(2-\beta J_{z}\right)}\right)^{n} .
\end{aligned}
$$

We note that by setting $n=0$ in Eq. (34a) we have

$$
\left\langle S^{2}\right\rangle=\frac{1}{2} N\left[2 /(2-\beta J)+1 /\left(2-\beta J_{z}\right)\right]
$$

and with Eq. (33b)

$$
\left\langle S_{x}^{2}\right\rangle=\left\langle S_{y}^{2}\right\rangle=\frac{1}{2} N /(2-\beta J) .
$$

The high-temperature spin fluctuations are all of order $N$ and behave mean-field-like near $T_{c}$ as was discussed in Sec. III. These may also be used to show that in the high-temperature regime the longitudinal and transverse factors of the static correlation functions decouple, e.g.

$$
\left\langle S_{z}^{2 n} S_{x}^{2}\right\rangle=\left\langle S_{z}^{2 n}\right\rangle\left\langle S_{x}^{2}\right\rangle
$$

Substituting Eq. (33a) and Eq. (34a) in Eq. (32), we obtain (after some simple sums)

$$
\begin{aligned}
S^{x x}(t)= & {\left[\left\langle S_{x}^{2}\right\rangle+i \frac{1}{2} \lambda t /\left(2-\beta J_{z}\right) \hbar\right] } \\
& \times e^{-(\lambda t)^{2} /\left(2-\beta J_{z}\right) \hbar^{2} N}
\end{aligned}
$$

which evidently is valid for $0 \leqslant t \leqslant t_{\max }$, where $t_{\max }$ $=\left(\hbar^{2} N / \lambda^{2}\right)^{1 / 2}$. Since $\left\langle S_{x}^{2}\right\rangle=O(N)$, for this range of time the time-correlation function valid to the leading order of $N$ is given by

$$
S^{x x}(t) \simeq\left\langle S_{x}^{2}\right\rangle e^{-(a N t)^{2}},
$$

where

$$
a_{N}(\lambda)=\left[\lambda^{2} / \hbar^{2} N\left(2-\beta J_{z}\right)\right]^{1 / 2} .
$$

The time-correlation function satisfies time-reversal symmetry. It is Gaussian, implying that the dynamic form factor is also Gaussian. ${ }^{18}$ Although our result [Eq. (36b)] applies equally to the $X Y$ like and Ising-like systems (i.e., $\lambda>0$ and $\lambda<0$, respectively) the temperature-dependent behavior of the "width" of the Gaussian factor $a_{N}^{-1}(\lambda)$ is markedly different between the two systems. For the Ising-like system, the width narrows as the critical temperature is approached from above and the system exhibits critical slow down.

For the $X Y$-like system, the situation is rather different. Here the static criticality is resolved from time, being contained $\operatorname{in}\left\langle S_{x}^{2}\right\rangle$ and not in the width $a_{N}^{-1}$. Hence, the time-correlation function for the $X Y$-like system cannot exhibit critical slow down. ${ }^{18}$ For the pure $X Y$ model (i.e., $J_{z}=0$ or $\lambda=J)$, the width factor reduces to $a_{N}{ }^{-1}=\left(\frac{1}{2} J^{2} /\right.$ $\left.\hbar^{2} N\right)^{-1 / 2}$ which has no temperature dependence at all at high temperatures $\left(\beta_{c} J \leqslant 2\right)$. This general form of the width, we believe, may be preserved if the number of interacting "near-neighbor" spins were reduced from $N$ to a small number, say $q$. In this case we have for the width $a_{q}{ }^{-1}$ $=\left(\frac{1}{2} q J^{2} / \hbar^{2}\right)^{-1 / 2}$, where we have restored the interaction strength from $J / N$ to $J$. [Recall that $N$ was introduced in Eq. (2) to keep the energy per spin finite in the limit $N \rightarrow \infty$.] It is interesting to note that a dynamic mean-field version of the threedimensional nearest-neighbor (nn) $X Y$ model for $T$ $>T_{c}$ gives exactly this form of the width for the timecorrelation function, where $q$ is the nn number. ${ }^{18}$ Also, the same form is obtained for the time-correlation function for the one-dimensional nn $X Y \bmod$ el..$^{9-11}$

\section{B. Low-temperature $X Y$ model}

We shall evaluate $\left\langle S_{z}^{2 n}\right\rangle$ and $\left\langle S_{z}^{2 n} S^{2}\right\rangle$ following the static analysis given in Sec. III B. For any integer $n$ we have

$$
\begin{aligned}
\left\langle S_{z}^{2 n}\right\rangle & =Z^{-1} \sum_{S} \sum_{S_{z}} g(S) S_{z}^{2 n} e^{-\beta \Psi C\left(S, s_{z}\right)} \\
& =\frac{2 n !}{n !}\left(\frac{N}{4 \beta \lambda}\right)^{n} .
\end{aligned}
$$

Hence, $\left\langle S_{z}^{2}\right\rangle=N / 2 \beta \lambda$. For the pure $X Y$ model, it becomes $\left\langle S_{z}^{2}\right\rangle=N / 2 \beta J$, which at the critical point $\beta J=\beta_{c} J=2$ assumes the high-temperature value, becoming independent of the temperature. In evaluating $\left\langle S_{z}^{2 n} S^{2}\right\rangle$, we recall that the maximum contributions of $S$ and $S_{z}$ come from widely separated regions of $N$ so that the two integrations have effectively two independent limits, i.e., the correlation function decouples,

$$
\left\langle S_{z}^{2 n} S^{2}\right\rangle=\left\langle S_{z}^{2 n}\right\rangle\left\langle S^{2}\right\rangle \text {. }
$$

We can directly evaluate $\left\langle S^{2}\right\rangle$ as, for example, in Eq. (37) giving

$$
\left\langle S^{2}\right\rangle=\left(\frac{1}{2} N \sigma\right)^{2}+\frac{N}{2 B}\left(\beta J \sigma+\frac{3+\sigma}{1+\sigma}\right)-\frac{2 N \sigma^{2}}{B^{2}\left(1-\sigma^{2}\right)^{2}},
$$

where we have used $\sigma$ and $B$ previously defined (see Sec. III B). It follows that we have

$$
\left\langle S_{x}^{2}\right\rangle=\frac{1}{2}\left[\left(\frac{1}{2} N \sigma\right)^{2}+\frac{N}{2 B}\left(\beta J \sigma+\frac{3+\sigma}{1+\sigma}\right)-\frac{2 N \sigma^{2}}{B^{2}\left(1-\sigma^{2}\right)^{2}}\right]-\frac{N}{4 \beta \lambda} .
$$

Observe that $\left\langle S_{x}^{2}\right\rangle=O\left(N^{2}\right)$ unlike in the high-temperature case [see Eq. (34c)]. The second-order terms shown in Eq. (40) are needed when evaluating the spin fluctuation since $\left\langle S_{x}\right\rangle=O(N)$.

Using Eq. (37), Eq. (38), and Eq. (40) in Eq. (32), we obtain for the time-correlation function 


$$
S^{x x}(t)=\left(\left\langle S_{x}^{2}\right\rangle+\frac{i t}{2 \hbar \beta}+\frac{t^{2}}{2 \hbar^{2} \beta^{2}}\right) e^{-\lambda t^{2} / \hbar^{2} \beta N},
$$

which evidently is valid for $0 \leqslant t \leqslant t_{\max }^{\prime}$ where $t_{\max }^{\prime}$ $=\left(\hbar^{2} \beta N / \lambda\right)^{1 / 2}$. Hence, for this range of time, we may take the correlation function to be

$$
\mathcal{S}^{x x}(t) \simeq\left\langle S_{x}^{2}\right\rangle e^{-\left(a_{N}^{\prime} t\right)^{2}}
$$

where $a_{N}^{\prime}=\left(\lambda / \hbar^{2} \beta N\right)^{1 / 2}$. It is again Gaussian, but the width is now temperature dependent. As the temperature is lowered, the width grows as $T^{-1 / 2}$. As the temperature is raised, the width narrows to a finite value. For the pure $X Y$ model the width has no discontinuity at $T_{c}$, i.e., for $\lambda=J, a_{N}^{\prime}=a_{N}$ at $\beta J=2$. As in the high-temperature side, the static criticality is resolved from time [see Eq. (41b)] so that there cannot be critical slow down in this time-correlation function as $T \rightarrow T_{c}$.

\section{Low-temperature Ising model}

For $\lambda$ negative, the evaluations of $\left\langle S_{z}^{2 n}\right\rangle$ and $\left\langle S_{z}^{2 n} S^{2}\right\rangle$ must be proceeded with more care as pointed out in Sec. III C. Writing $\lambda=-\lambda^{\prime}$ (where $\lambda^{\prime}$ is a positive quantity), we have for any integer $n$

$$
\begin{array}{rl}
\left\langle S_{z}^{2 n}\right\rangle=Z^{-1} \sum_{S} & g(S) e^{\beta J S^{2} / N} \\
& \times\left(\sum_{S_{z}} S_{z}^{2 n} e^{B \lambda^{\prime} S_{z}^{2} / N}\right) .
\end{array}
$$

The term inside the parentheses in Eq. (42a) may be handled as follows:

$$
\begin{aligned}
\sum_{S_{z}} S_{z}^{2 n} e^{a s_{z}^{2}} & =\left(\frac{\partial}{\partial a}\right)^{n} \sum_{S_{z}} e^{a S_{z}^{2}} \\
& =\left(\frac{\partial}{\partial a}\right)^{n}\left[2 a^{-1 / 2} e^{a s^{2}} D\left(a^{1 / 2} S\right)\right] \\
& \simeq a^{-1} e^{a S^{2}} S^{2 n-1}\left(1-\frac{2 n-1}{2 a S^{2}}+\cdots\right),
\end{aligned}
$$

where $a=\beta \lambda^{\prime} / N, D$ is Dawson's integral [see Eq. (20)], and in obtaining Eq. (42b) the asymptotic expansion of Dawson's integral [Eq. (21)] has been used.

Substituting Eq. (42b) in Eq. (42a), we finally obtain

$$
\left\langle S_{z}^{2 n}\right\rangle=\left(\frac{1}{2} N \sigma\right)^{2 n}\left[1-\frac{2}{N \sigma}\left(2 n A-\frac{n(2 n-1)}{B_{z} \sigma}\right)+O\left(N^{-2}\right)\right],
$$

where

$$
A=\frac{1}{B_{z}(1+\sigma)}+\frac{2 \sigma}{B_{z}^{2}\left(1-\sigma^{2}\right)^{2}}-\frac{1}{\beta \lambda^{\prime} \sigma}-\frac{\beta J}{2 B_{z}},
$$

and see Eq. (24b) for $B_{z}$.

Observe that for $n=1$ we have

$$
\left\langle S_{z}^{2}\right\rangle=S_{0}^{2}\left[1+O\left(N^{-1}\right)\right],
$$

where we recall that $S_{0}=\frac{1}{2} N \sigma$. The terms of order $N^{-1}$ in Eq. (43b) thus determine the spin fluctuations $Y^{z z}=\left\langle S_{z} S_{z}\right\rangle-\left\langle S_{z}\right\rangle^{2}$. We note that $Y^{z z}$ are $O(N)$ and have critical behavior. In evaluating the timecorrelation function $\oint^{x x}(t)$ with Eq. (43a), the terms of order $N^{-1}$, which also depend on $n$, will give additional time-dependent factors. To be consistent with the spirit of our expansion (i.e., an expansion in powers of $N^{-1}$ ), we must restrict largest values of $n$ to be less than $O\left(N^{1 / 2}\right)$.

Similarly, we obtain for any integer $n$,

$$
\left\langle S_{z}^{2 n} S_{x}^{2}\right\rangle=S_{0}^{2 n} N / 2 \beta \lambda^{\prime} .
$$

Observe that by setting $n=0$ in Eq. (44), we get $\left\langle S_{x}^{2}\right\rangle=O(N)$. It is linear in $N$, whereas the leading term of $\left\langle S_{z}^{2}\right\rangle$ is quadratic in $N$. The spin fluctuations $Y^{x x}$ have no anomalous behavior at $T_{c}$.

Substitution of Eqs. (43a) and (44) in Eq. (30b) gives the following expression for the time-correlation function:

$$
\begin{aligned}
\mathcal{S}^{x x}(t)= & \left(\frac{N}{2 \beta \lambda^{\prime}}-\frac{i t \lambda}{2 \hbar}\left(A \sigma-B_{z}^{-1}\right)\right) \cos \left(\frac{\lambda \sigma}{\hbar}\right) t \\
& +\frac{1}{2} i\left(\frac{1}{2} N \sigma-A-\frac{t^{2} \lambda^{2} \sigma}{2 B_{2} \hbar^{2}}\right) \sin \left(\frac{\lambda \sigma}{\hbar}\right) t .
\end{aligned}
$$

In the above expression, those containing $t$ and $t^{2}$ come from the terms of $N^{-1}$. Hence, our results are valid for $0 \leqslant t<t_{\max ^{\prime}}$ where $t_{\max }=\left(\hbar^{2} N / \lambda^{2}\right)^{1 / 2}$. In this range of $t$, the correlation function is

$$
\mathcal{S}^{x x}(t) \approx\left\langle S_{x}^{2}\right\rangle \cos (\lambda \sigma / \hbar) t+\frac{1}{2} i\left\langle S_{z}^{2}\right)^{1 / 2} \sin (\lambda \sigma / \hbar) t
$$

In the low-temperature region $\left(T<T_{c}\right)$ the correlation function is oscillatory, whereas in the high-temperature region the correlation function shows Gaussian behavior. Since $\sigma \rightarrow 0$ as $T \rightarrow T_{c}-$, it is evident that the Ising-like system exhibits critical slow down as in the high-temperature side. The form of critical slow down is, however, very different from the conventional form. ${ }^{23}$ Finally, we note that in the isotropic limit (i.e., $\lambda \rightarrow 0$ ) both the $X Y$ - and Ising-correlation functions, in Eqs. $(41 \mathrm{~b})$ and $(45 \mathrm{~b})$ respectively, reduce to the same time-independent correlation function as is required.

\section{CONCLUDING REMARKS}

We have derived the transverse component of the time-correlation function $S^{x x}(t)$ for the spin Van der Waals system, valid to the time scale $t<O\left(N^{1 / 2}\right)$ in an appropriate choice of units. Our results for the time-correlation function are not of exponential form as one might have expected from the similarity between the Van der Waals and OrnsteinZernicke-type models. 
As is already known, all the Van der Waals systems belong to one static universality class quite unlike the nearest-neighbor models. Our work shows that dynamic properties are, however, more subtle: For $T>T_{c}$ there is one dynamic class, but for $T<T_{c}$ there are two classes.

That there is but one static universality class for the Van der Waals system seems nevertheless reasonable. In the high-density limit, the spin interaction evidently is smoothed out, thereby masking spin dimensionality, at least to first order. But for dynamics, time evolution is still governed by the commutation relations of spin operators which are not affected by high densities. As a result, apparently not all subtle details of quantum origin are averaged out. For example, for the Ising-like system in the condensed phase, $\oint^{x x}(t)$ shows a kind of Larmor precession about the axis of the spontaneous magnetization $\left\langle S_{z}\right\rangle$. It precesses indefinitely and has a frequency which depends on the spontaneous magnetization. At high temperatures, the system ceases to precess as there is no longer any spontaneous magnetization. For the
$X Y$-like system in the condensed phase, the same correlation function cannot precess as in the Isinglike system, since the spontaneous magnetization now is also in the transverse direction. Thus, the time-correlation function smoothly approaches an equilibrium and it is also unaffected by the onset of critical fluctuations. Finally, since these dynamic features are consequences of the commutativity property of spin operators, it seems reasonable to conjecture that the nearest-neighbor models would have qualitatively similar dynamic behavior as described by the Van der Waals models.

\section{ACKNOWLEDGMENTS}

We are indebted to Professor L. P. Bouckaert for his continuing and stimulating interest in the progress of our work. This research is supported in part by DOE ERDA under the Contract No. EG-77-S09-1023. We also thank NATO for travel support (Grant No. 1024) which has made it possible for us to carry out our collaborative work.
${ }^{1} Y$. de Ribaupierre and F. D. Manchester, J. Phys. C 7, 2126 (1974).

${ }^{2}$ C. Kittel and H. Shore, Phys. Rev. 138, A1165 (1965). ${ }^{3}$ Th. Niemeijer, Physica (Utr.) 48, $\overline{467}$ (1970).

${ }^{4}$ G. Vertogen and A. S. DeVries, Physica (Utr.) 59, 634 (1972).

${ }^{5}$ For these cooperative models with short-range interaction, it is now well established that static universality classes are determined by relevant variables, one of which is spin dimensionality. [See, for example, H. E. Stanley, Introduction to Phase Transitions and Critical Phenomena (Oxford U. P., Oxford, 1971)]. Thus, mean-field models evidently require much weaker static universality conditions.

${ }^{6}$ B. I. Halperin and P. C. Hohenberg, Phys. Rev. 177, 952 (1969).

${ }^{7}$ This work is also suggested by an earlier work on the dynamic behavior of the $S=\frac{1}{2}$ nearest-neighbor $X Y$ model. See M. H. Lee, Phys. Rev. B 8, 3290 (1973). It was shown there that under a high-frequency approximation the nearest-neighbor $X Y$ model becomes dynamically equivalent to the constant-coupling $X Y$ model.

${ }^{8}$ For the Ising-like case, our results given here may be regarded complementary to the longitudinal correlation function for $T \gtrsim T_{c}$ obtained by, e.g., K. Kawasaki and T. Yamada [Prog. Theor. Phys. 39, 1 (1968)], where the spin dynamics is generated by a master equation (i.e., a kinetic Ising model). The dynamic critical properties of such a stochastic system and other related ones are extensively reviewed recently by P. C. Hohenberg and B. I. Halperin [Rev. Mod. Phys. 49, 435 (1977)].

${ }^{9}$ A. Sur, D. Jasnow, and I. J. Lowe, Phys. Rev. B $\underline{12}$, 3845 (1975)

${ }^{10} \mathrm{U}$. Brandt and K. Jacoby, Z. Phys. B 25, 181 (1976).

${ }^{11} \mathrm{H}$. W. Capel and J. H. H. Perk, Physica (Utr.) 87A, 211 (1977).

${ }^{12}$ J. H. Van Vleck, Electric and Magnetic Susceptibilities
(Clarendon, Oxford, 1932), pp. 323 and 324.

${ }^{13}$ R. H. Brout, Phase Transitions (Benjamin, New York, 1965), p. 11.

${ }^{14}$ That is, the entropy per spin $S_{e} / N=-k W(S)+k \ln 2$ $+\mathrm{O}\left(N^{-1}\right)$. Hence, $-k W(S)$ may be identified as our spin-dependent entropy term.

${ }^{15}$ A. Erdélyi, Asymptotic Expansions (Dover, New York, $1956)$, p. 36.

${ }^{16}$ Recall that $S$ is obtained by vector addition of individual spins, i.e., $\overrightarrow{\mathrm{s}}=\sum_{i=1}^{N} \overrightarrow{\mathrm{s}}_{i}$ where $s_{i}=\frac{1}{2}$ for all $i$, and $S_{0}$ is one of the possible values of $S$. Evidently min $S$ can result when individual spins are in a disordered state, whereas $\max S$ can result when individual spins are in an ordered state. Observe that $-W(S) \rightarrow 0$ as $S / N \rightarrow 0$ and $-W(S)<0$ for $0<S / N<\frac{1}{2}$ [see Eq. (8)]

${ }^{17}$ The specific heat vanishes to the order $\mathrm{O}\left(N^{-1}\right)$. For mean-field models the specific heat has a discontinuity at $T_{c}$.

${ }^{18}$ See M. H. Lee in Ref. 7.

${ }^{19}$ Near $T_{c}$, Eq. (16c) gives the familiar mean-field result $\sigma \sim\left(\beta / \beta_{c}-1\right)^{1 / 2}$.

${ }^{20} \mathrm{M}$. Abramowitz and I. A. Stegun, Handbook of Mathematical Functions Natl.Bur. of Stds., Appl. Math. Ser. No. 55, (U. S. GPO, Washington, D.C., 1970), p. 298; Higher Transcendental Functions, Vol. II, edited by A. Erdélyi (McGraw-Hill, New York, 1953), p. 147.

${ }^{21}$ This result and also the similar one for the $X Y$-like systems may be compared with the numerical work of D. Jasnow and M. Wortis [Phys. Rev. 176, 739, (1968)] for the nearest-neighbor anisotropic classical Heisenberg model. For the nearest-neighbor model, actually the exponent appears to behave universally but not the critical temperature.

${ }^{22}$ For $n n$ models, one has similar equations of motion, which are examples of the generalized Langevin equation. These equations would be highly nonlinear and would not ordinarily be readily soluble except in some special limits. [See M. H. Lee and R. Dekeyser, 
Physica (Utr.) 86-88 B +C, 1273 (1977), and references therein.] For Van der Waals models, however, the nonlinear or "random force" terms [the quadratic terms in Eq. (26)] are nonregenerative so that exact general solutions of the time evolution can be obtained. ${ }^{23}$ See, for example, W. Marshall and R. D. Lowde, Rep. Prog. Phys. 31, 705 (1968). 